\title{
Ultrafast harmonic rf kicker design and beam dynamics analysis for an energy recovery linac based electron circulator cooler ring
}

\author{
Yulu Huang \\ Institute of Modern Physics, Chinese Academy of Sciences, Lanzhou 730000, China; \\ Thomas Jefferson National Accelerator Facility, Newport News, Virginia 23606, USA; \\ and University of Chinese Academy of Sciences, Beijing 100049, China \\ Haipeng Wang, ${ }^{*}$ Robert A. Rimmer, Shaoheng Wang, and Jiquan Guo \\ Thomas Jefferson National Accelerator Facility, Newport News, Virginia 23606, USA
}

(Received 11 June 2016; published 22 August 2016)

\begin{abstract}
An ultrafast kicker system is being developed for the energy recovery linac (ERL) based electron circulator cooler ring (CCR) in the proposed Jefferson Lab Electron Ion Collider (JLEIC, previously named MEIC). In the CCR, the injected electron bunches can be recirculated while performing ion cooling for 10-30 turns before the extraction, thus reducing the recirculation beam current in the ERL to $1 / 10-1 / 30$ $(150 \mathrm{~mA}-50 \mathrm{~mA})$ of the cooling beam current (up to $1.5 \mathrm{~A}$ ). Assuming a bunch repetition rate of $476.3 \mathrm{MHz}$ and a recirculating factor of 10 in the CCR, the kicker is required to operate at a pulse repetition rate of $47.63 \mathrm{MHz}$ with pulse width of around $2 \mathrm{~ns}$, so that only every 10th bunch in the CCR will experience a transverse kick while the rest of the bunches will not be disturbed. Such a kicker pulse can be synthesized by ten harmonic modes of the $47.63 \mathrm{MHz}$ kicker pulse repetition frequency, using up to four quarter wavelength resonator (QWR) based deflecting cavities. In this paper, several methods to synthesize such a kicker waveform will be discussed and a comparison of their beam dynamics performance is made using ELEGANT. Four QWR cavities are envisaged with high transverse shunt impedance requiring less than $100 \mathrm{~W}$ of total rf power for a Flat-Top kick pulse. Multipole fields due to the asymmetry of this type of cavity are analyzed. The transverse emittance growth due to the sextupole component is simulated in ELEGANT. Off-axis injection and extraction issues and beam optics using a multicavity kick-drift scheme will also be discussed.
\end{abstract}

DOI: 10.1103/PhysRevAccelBeams.19.084201

\section{INTRODUCTION}

Cooling of ion beams will be a critical technology in delivering high luminosities over a broad center of mass (CM) energy range in the proposed JLEIC [1,2]. The present JLEIC design envisages an energy recovery linac (ERL)-based high-energy (up to $55 \mathrm{MeV}$ ) magnetized and bunched electron beam cooler in the collider ion ring to cool the ions at energies of up to $100 \mathrm{GeV} / \mathrm{u}$, as shown in Fig. 1 (solid black), with the option of adding a recirculation ring (dash green) as a future upgrade.

In the upgrade scheme, each electron bunch will recirculate 10-30 turns in the circulator ring until the beam needs to be refreshed. The used bunch will be extracted to the ERL with 180 degree rf phase shift for energy recovery and finally be sent to a dump, while the recovered energy is used to accelerate a new incoming bunch. The new bunch will be kicked on axis and the recirculation will continue.

\footnotetext{
*haipeng@jlab.org

Published by the American Physical Society under the terms of the Creative Commons Attribution 3.0 License. Further distribution of this work must maintain attribution to the author $(s)$ and the published article's title, journal citation, and DOI.
}

With the recirculation scheme, the bunch repetition rate and the beam current in the ERL can be reduced by a factor of 10-30 (equal to the number of circulations) compared to the beam in the circulator ring, which greatly eases the technical challenges of the high current magnetized electron source, high order modes damping in the ERL and high power at the beam dump [3-5].

A critical component in this scheme is the ultrafast kicker needed to periodically deflect individual electron bunches in and out of the circulator ring from and to the driver ERL, while leaving the adjacent bunches in the orbit of the circulator ring undisturbed. To avoid disrupting the

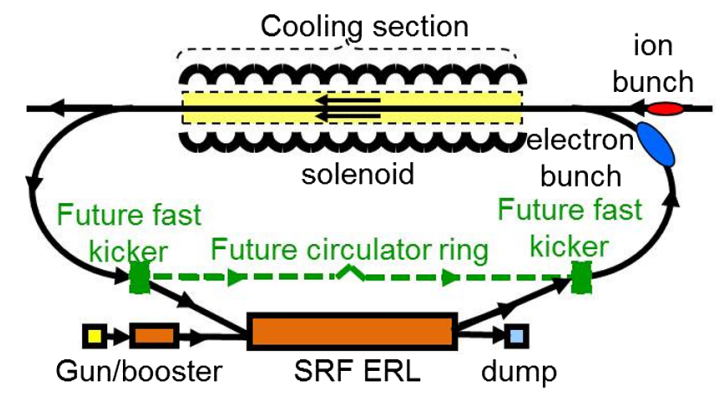

FIG. 1. Schematic of ERL-based bunched beam cooler (black solid) with option for future recirculation path (green dash). 

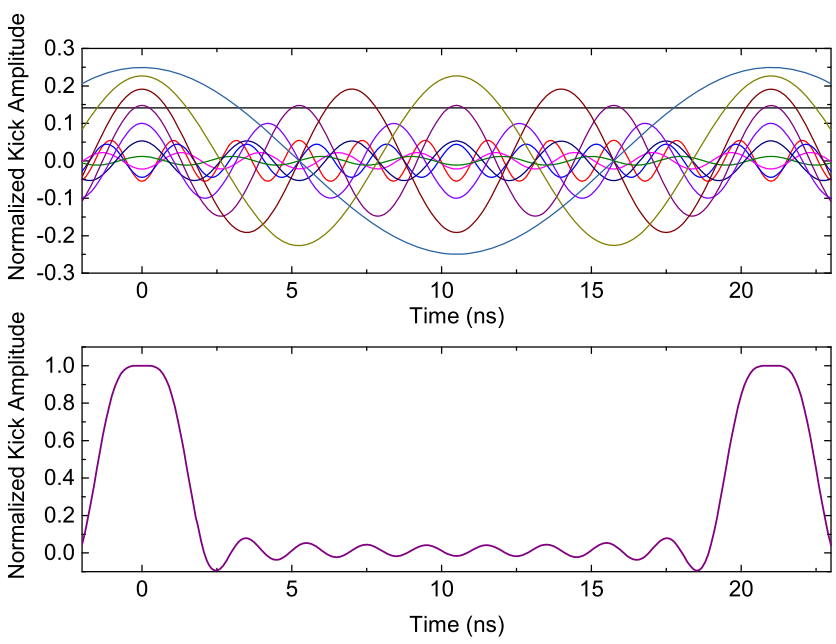

FIG. 2. Normalized continuous kicker waveform of $47.63 \mathrm{MHz}$ repetition rate (bottom) resulting from the summation of a DC offset and 10 harmonics of the base frequency of $47.63 \mathrm{MHz}$ (top).

recirculating bunches near the kicked bunch, the rise-andfall time of the kicker pulse needs to be very short (less than the bunch spacing of $2.1 \mathrm{~ns}$ for a $476.3 \mathrm{MHz}$ bunch train). The kicker pulse repetition rate needs to be the same as the ERL bunch repetition rate, which will be $47.63 \mathrm{MHz}$ for a recirculation factor of 10 . These specifications, high repetition rate and fast rise-and-fall time combined together, are well beyond the state-of-art of traditional pulsed magnet kickers [6].

Several solutions have been explored to pursue these technologies. A beam-beam kicker for super-fast bunch handling has already been demonstrated [7], which adopts a high pulse current, low energy beam to produce the deflecting force, and provides a kick duration of less than a nanosecond. The ultimate limitation of this beam-based kicker is the high charge source of the low energy beam, which is limited by space charge effects.

In this paper, we will discuss an efficient rf-based kicker concept proposed to produce the pulse using harmonic rf cavities. These cavities will simultaneously resonate at multiple harmonic frequencies and be excited with appropriate amplitudes and phases that correspond to the Fourier components of a periodic narrow pulse. This effectively will synthesize a continuous waveform of short pulses [8,9], as shown in Fig. 2. These rf kicker cavities will run continuously to provide a cooling beam to counteract emittance growth while colliding. The frequencies, phases, and relative amplitudes of each harmonic must be controlled precisely to maintain the pulse-to-pulse stability during the injection, recirculation and extraction of all bunches in the CCR.

\section{HARMONIC KICKER WAVEFORM SYNTHESIZATION}

Similar concepts using a combination of a series of harmonic modes have already been explored for other

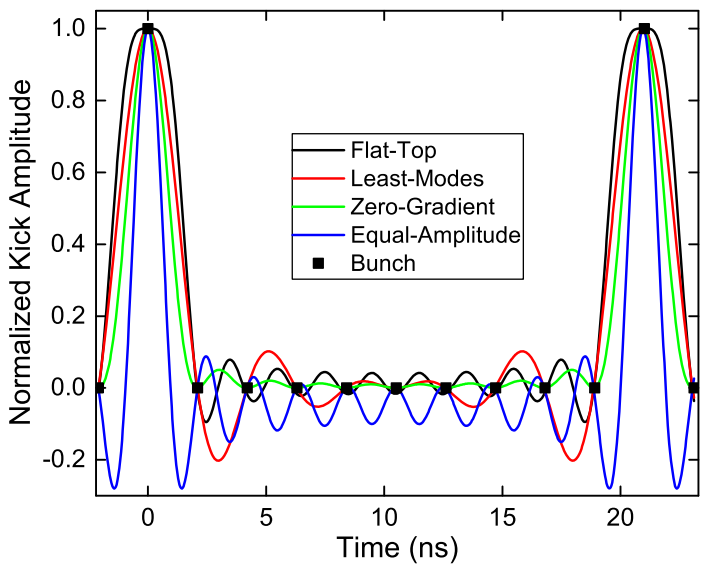

FIG. 3. Four different synthesized kick waveform schemes for a recirculating factor of 10 and bunch repetition frequency of 476.3 MHz.

applications, such as in the compact noninvasive electron bunch-length monitor [10], the multiharmonic impulse cavity [11], the multifrequency high gradient accelerator structure [12], and the stripline kicker [13]. There are several mathematical solutions to approximate the ideal kick waveform with a finite number of rf harmonics. In this study four schemes are explored to determine the amplitudes and phases of these harmonics, which result in four different kicker waveforms, as shown in Fig. 3.

The flat-top scheme comes directly from the fast Fourier transform of periodic rectangular pulses. The Fourier series has an infinite number of elements, the amplitudes and phases of which are related to the width and amplitude of the original square pulse. The frequencies of these elements start from $47.63 \mathrm{MHz}$ and increase in positive integer multiples. Reconstructing the pulse with a DC component and only the first 10 harmonics (from $47.63 \mathrm{MHz}$ to 476.3 MHz), and adjusting the harmonic amplitudes to achieve the best pulse uniformity within $+/-3 \sigma$ of the bunch length, produces a flat-top kicking pulse with the most uniform kick for the kicked bunch and zero kick at the centroid of the recirculating bunches. However, this waveform fluctuates in between the kicking pulses such that there will be a residual slope and head-tail distortion of the recirculating bunches [14].

The zero-gradient scheme is synthesized by setting constraints of zero amplitude and zero gradient at these intermediate bunch positions, which leaves these bunches undisturbed during circulation. However, the kicking pulse is less-uniform for the kicked bunch $[15,16]$, leading to some emittance growth on injection.

The least-modes scheme is synthesized by removing the gradient constraints from the zero-gradient scheme, which will reduce the harmonic modes number by half. This actually provides a more uniform kicking pulse but introduces a larger slope at the intermediate positions between kicking pulses $[14,16]$. 


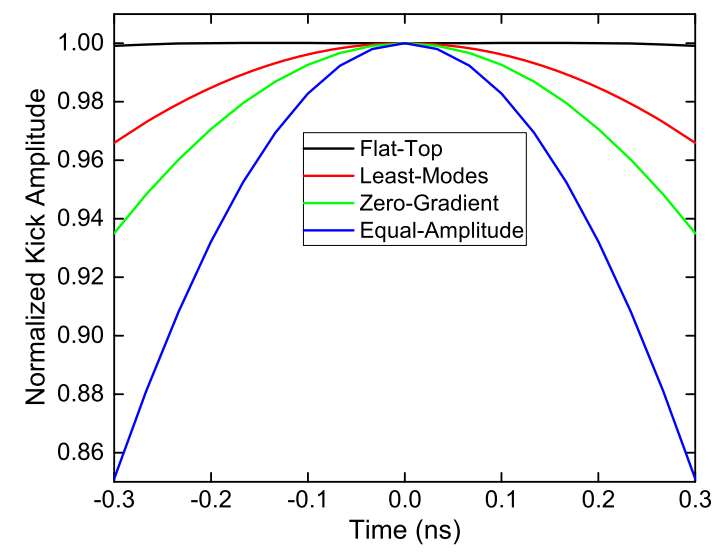

FIG. 4. Comparison of the kicking pulse curvature over $+/-3 \sigma$ of $3 \mathrm{~cm}$ kicked electron bunch for the four waveform schemes with a recirculating factor of 10 .

The equal-amplitude scheme has every harmonic with equal amplitude and no DC offset but has the largest kicking pulse curvature and large slopes at the intermediate positions [9].

Figure 4 shows a comparison of the kicking pulse curvature over $+/-3 \sigma$ of a $3 \mathrm{~cm}$ (rms) electron bunch. The nonuniformity of the kicking pulse can be defined by the percentage difference between the peak amplitude of the pulse at the center of the bunch and the pulse amplitude head and tail of the full bunch length. In this 10 turn circulation scheme, the nonuniformity is less than $0.01 \%$ for the flat-top scheme, $3.5 \%$ for the least-modes scheme, $6.4 \%$ for the zero-gradient scheme and $15 \%$ for the equalamplitude scheme. The nonuniform kicking pulse will have an adverse effect on the transverse beam quality of the kicked bunch and increase the transverse emittance, which will be discussed in the following sections.

Table I summarizes the normalized kick voltages for each harmonic in the different waveform schemes, in which the positive values means that harmonic modes are

TABLE I. Normalized kick amplitude for each harmonic.

\begin{tabular}{lrccc}
\hline \hline $\begin{array}{c}\text { Mode } \\
\text { (MHz) }\end{array}$ & Flat-top & Zero-gradient & Least-modes & Equal-amplitude \\
\hline 47.63 & 0.249 & 0.180 & 0.200 & 0.100 \\
95.26 & 0.227 & 0.160 & 0.200 & 0.100 \\
142.89 & 0.192 & 0.140 & 0.200 & 0.100 \\
190.52 & 0.148 & 0.120 & 0.200 & 0.100 \\
238.15 & 0.100 & 0.100 & 0.100 & 0.100 \\
285.78 & 0.053 & 0.080 & $\ldots$ & 0.100 \\
333.41 & 0.012 & 0.060 & $\ldots$ & 0.100 \\
381.04 & -0.022 & 0.040 & $\ldots$ & 0.100 \\
428.67 & -0.044 & 0.020 & $\ldots$ & 0.100 \\
476.3 & -0.055 & $\ldots$ & $\ldots$ & 0.100 \\
DC & 0.140 & 0.100 & 0.100 & $\ldots$ \\
Total & 1.000 & 1.000 & 1.000 & 1.000 \\
\hline \hline
\end{tabular}

stimulated at 0 phase and negative ones at $\pi$ phase. The total kick voltage is

$$
V_{t}=V_{0}+\sum_{n=1}^{N} V_{t n} \cos \left(n \omega_{1} t+\varphi_{n}\right) \text {. }
$$

Here the constant term $V_{0}$ represents a DC offset, $\omega_{1}$ is the bunch repetition frequency in the ERL (47.63 MHz), $N$ is the harmonic number, and $\varphi_{n}$ is the rf phase of the $n$th harmonic.

\section{BEAM DYNAMICS TRACKING SIMULATION IN ELEGANT}

To make a comparison of different waveform schemes, the effects on transverse beam quality for both kicked and unkicked bunches were simulated using ELEGANT [17]. In the simulation, a single round electron bunch with Gaussian distribution is generated for the 10-turn recirculation. Parameters used in the simulation are summarized in Table II.

The tracking scheme in ELEGANT is shown in Fig. 5. In the simulation, a single electron bunch with an initial positive transverse momentum (equivalent to an angle of $1 \mathrm{mrad}$ ) receives a negative kick from the injection kicker (Kicker 1), and is deflected onto the design orbit of the CCR. After 10 turns of recirculation, the bunch receives another negative kick from the extraction kicker (Kicker 2) and gets ejected back to the ERL. Every bunch in the ERL (47.63 MHz repetition rate) will be kicked into the CCR, and only every 10th bunch in the CCR $(476.3 \mathrm{MHz}$ repetition rate) will be kicked out to the ERL. The circulator ring was approximated by a one turn linear transfer matrix; the kicker waveform was generated using a series of zerolength rf deflectors with appropriate frequencies, phases, and amplitudes that are combined at a single point; the DC component is simplified as a zero frequency rf deflector with the appropriate phase and amplitude [15]. In reality, this DC voltage can be replaced by a steering magnet.

The rf phase difference between two kickers and the path length of the cooler ring L1 (Fig. 5 upper) is adjusted so

TABLE II. Input parameters in ELEGANT simulation.

\begin{tabular}{lcc}
\hline \hline Parameter & Value & Unit \\
\hline Electron energy & 55 & $\mathrm{MeV}$ \\
RMS bunch length & 3 & $\mathrm{~cm}$ \\
Bunch distribution & Gaussian & \\
Bunch frequency & 476.3 & $\mathrm{MHz}$ \\
Normalized transverse emittance & 1.074 & $\mathrm{~mm} \mathrm{mrad}$ \\
Normalized vertical emittance & 1.074 & $\mathrm{~mm} \mathrm{mrad}$ \\
Energy spread & $3 \mathrm{E}-4$ & \\
Recirculation turns & 10 & $\mathrm{No}$. \\
Kick angle & 1 & $\mathrm{mrad}$ \\
Total kick voltage & 55 & $\mathrm{kV}$ \\
\hline \hline
\end{tabular}




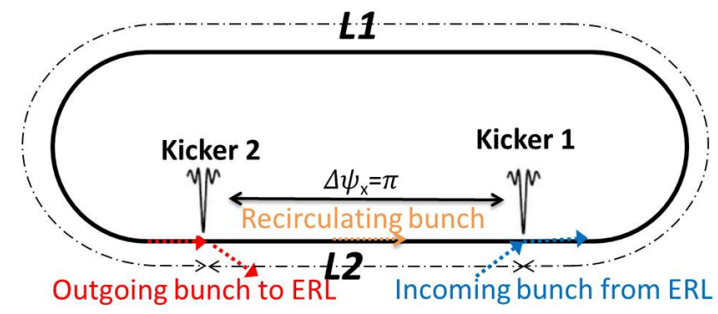

FIG. 5. Two kickers tracking scheme in ELEGANT. Kicker 1 is for injection and Kicker 2 is for extraction.

that the recirculating bunch will not be kicked out by Kicker 2 in the first 9 turns, and is finally ejected in the last turn. The path length of the recirculation path L2 (Fig. 5 lower) is adjusted to make sure the recirculating bunches experience the same waveform slope at both kicker locations. The bunch distortion due to the wave slopes between kicking pulse can be totally canceled out by setting the betatron phase advance between the two kickers to be exactly 180 degrees (or its odd multiples) [14]. The total ring length must therefore satisfy the following equation:

$$
L 1+L 2=(10 N \pm \delta) \lambda_{10}
$$

Here 10 is the recirculation factor, $N$ is a positive integer, which will determine the total length of ring, for example, $N=30$ will result to a total ring length of about $190 \mathrm{~m} . \lambda_{10}$ is the wavelength of the bunch repetition frequency (476.3 MHz), which is also the bunch separation distance $(0.63 \mathrm{~m})$ in the cooler ring. $\delta$ is the bunch spacing number in the waveform that the bunch walks per turn relative to the kicker position, which should not be the co-prime with the recirculation turn number, in this 10-turn case

$$
\delta= \pm 1, \pm 3, \pm 7, \pm 9
$$

In the simulation we used $\delta=1$, so that only 1 bunch space is being walked per turn, as is shown in Fig. 6 (left),
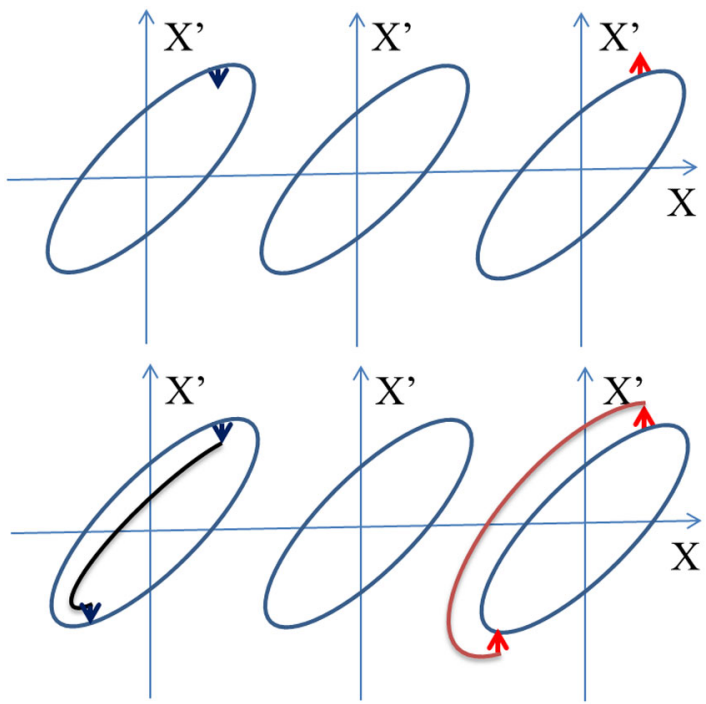

FIG. 7. The cancellation scheme of the residual kick with two identical kickers. A single particle in the phase space, receives two identical residual kicks with 180 degrees phase advance in $\mathrm{X}-\mathrm{X}$ ' plane, and returns to its original phase space orbit.

the number in the figure indicates the recirculation turn number. Figure 6 also shows the case of $\delta=3$ (right).

The cancellation of the residual kick with two identical kickers is demonstrated in Fig. 7. For a single particle in a bunch, a transverse kick will change its transverse momentum. As the particle proceeds along the orbit, the transverse momentum and position follow a betatron oscillation. If the two kickers are separated in a distance with 180 degree (or its odd multiples) betatron phase advance, and the rf phases of the two kickers are set in such a way that the recirculation bunches experience the same wave slope at the two kicker positions, then these particles in the bunch that experience a small residual kick from the extraction kicker (Kicker 2) will experience the same kick from injection kicker (Kicker 1), and return to the original phase space orbit. In this way, the emittance is preserved locally even with nonuniform residual kick voltage variations along the bunch.
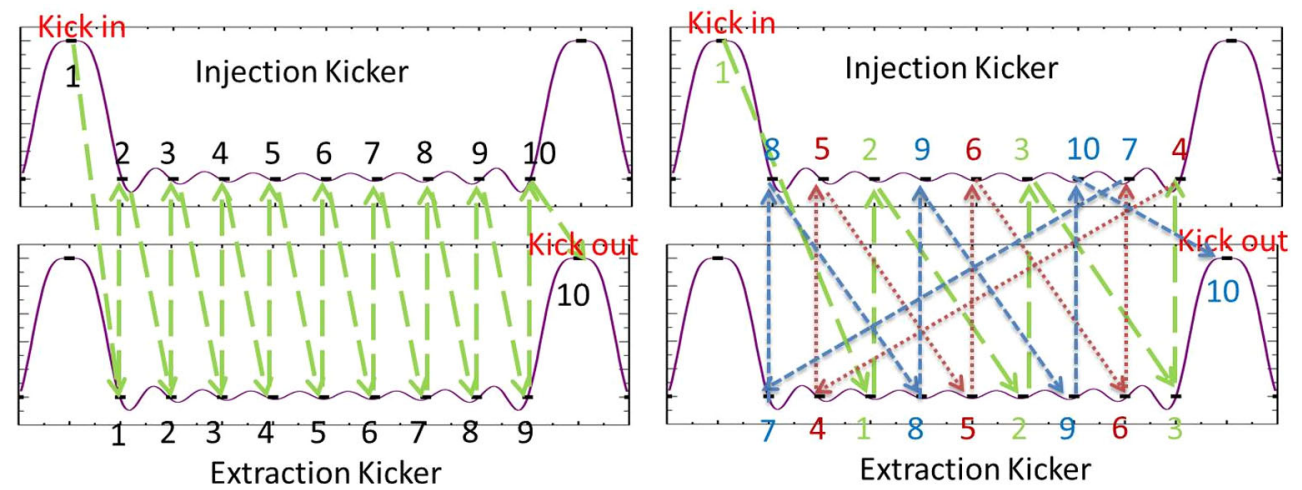

FIG. 6. Kicker scheme for 10-turn recirculation with two identical kickers, the alternating bunch kick is in one bunch spacing (left) and three bunch spacing (right). 


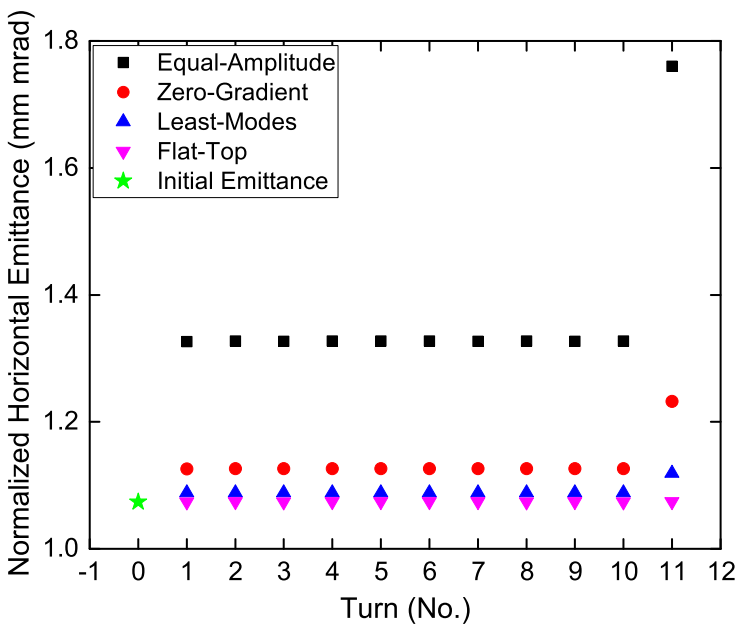

FIG. 8. Turn-by-turn normalized horizontal emittance for a single electron bunch in the two kicker scheme with 180 degrees betatron phase advance in between. The monitor is placed immediately after the injection kicker for the first 10 turns and after the extraction kicker for the last turn.

With the cancellation of the residual kick voltage between two identical kickers, negligible emittance growth has been seen during 10 turn recirculation for all kicker waveform schemes, as shown in Fig. 8. The emittance growth mainly comes from the injection kicker when the bunch is first kicked into the CCR, and from the extraction kicker when the bunch is finally kicked out to the ERL. The growth proportions of these four schemes are listed in Table III, and are related to the curvature of the kicking pulse as already compared in Fig. 4.

The flat-top scheme has the minimum emittance growth both at injection and at extraction due to the most uniform kicking pulse. The least-modes scheme is almost as good as the flat-top scheme with only half the number of harmonic modes when kicked in and during the circulation. A larger emittance growth occurs when the bunch is kicked out to the ERL, but will no longer affect the cooling performance. The zero-gradient scheme and the equal-amplitude scheme have relatively large emittance during the whole cooling period and especially when the bunch is extracted.

If necessary the emittance growth due to the kicking pulse curvature could be compensated by introducing another two identical kickers, one before the injection kicker with a betatron phase advance of $\Delta \psi_{x}=\pi$ and the

TABLE III. Emittance growth for different kicking waveform schemes.

\begin{tabular}{lcc}
\hline \hline & Injection & Extraction \\
\hline Flat-top & $0.03 \%$ & $0.03 \%$ \\
Least-modes & $1.5 \%$ & $4.4 \%$ \\
Zero-gradient & $5.2 \%$ & $15.8 \%$ \\
Equal-Amplitude & $25.3 \%$ & $68.6 \%$ \\
\hline \hline
\end{tabular}

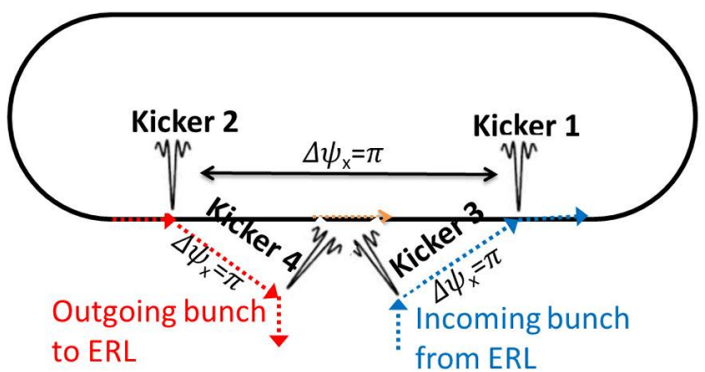

FIG. 9. Compensation scheme for the nonuniform kicking pulse by using identical predistortion kicker (Kicker 3) and postdistortion kicker (Kicker 4).

other one followed after the extraction kicker with the same betatron phase advance, as shown in Fig. 9. With the predistortion and postdistortion correction in the phase space, the distortion of the electron bunch due to the kicking pulse curvature is totally removed and the emittance performance of the least-mode scheme, zero-gradient scheme and the equal-amplitude scheme is almost the same as the flat-top scheme. If the beam quality after the recirculation can be ignored, the Kicker 4 can be removed from Fig. 9, the tracking result with three kickers (Kicker 1, Kicker 2, and Kicker 3) is shown in Fig. 10.

All above discussion is in the ideal situation, the beam optics are perfect thus the 180 degree betatron phase advance is exact, the $\mathrm{rf}$ frequencies, phases and amplitudes of all modes are precisely controlled thus the kicking pulse at two kickers are identical and the recirculating bunches will drop on two identical wave slopes to get a complete cancellation.

But in the actual operation, there are many systematic and random errors, such as the manufacturing errors on the beam optics device, the different phase advance experienced by different particles in the bunch due to the energy

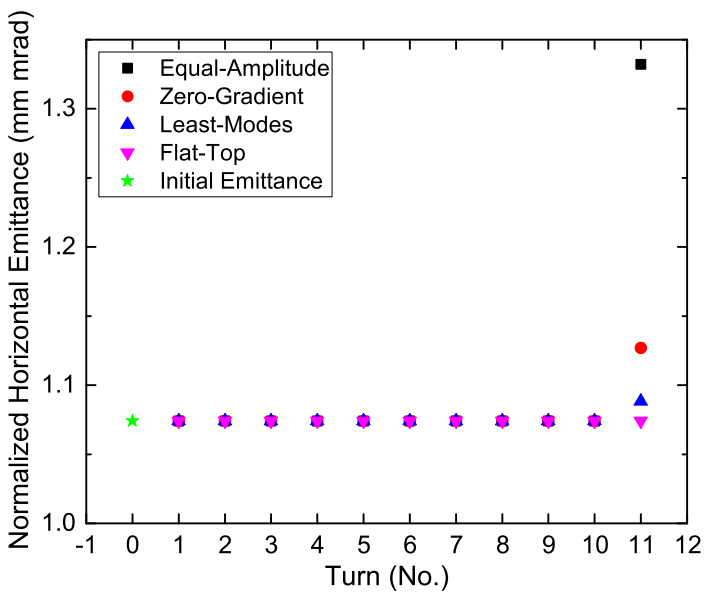

FIG. 10. Turn-by-turn normalized horizontal emittance growth for a single electron bunch with a predistortion kicker to compensate the nonuniform kicking pulse but no postdistortion kicker. 
TABLE IV. Calculated relative emittance growth due to errors in the beam optics and rf control system.

\begin{tabular}{|c|c|c|c|c|c|c|}
\hline & \multicolumn{2}{|c|}{ Betatron phase errors } & \multicolumn{2}{|c|}{ Voltage amplitude errors } & \multicolumn{2}{|c|}{ Time delay errors } \\
\hline & $+5 \%$ & $-5 \%$ & $+5 \%$ & $-5 \%$ & $+5 \%$ & $-5 \%$ \\
\hline Flat-top & $3.73 \%$ & $5.33 \%$ & $0.75 \%$ & $0.81 \%$ & $5.25 \%$ & $4.70 \%$ \\
\hline Least-mode & $20.08 \%$ & $16.23 \%$ & $2.71 \%$ & $2.64 \%$ & $2.63 \%$ & $2.25 \%$ \\
\hline Zero-gradient & $0.19 \%$ & $0.36 \%$ & $0.01 \%$ & $0.13 \%$ & $1.60 \%$ & $0.80 \%$ \\
\hline Equal-amplitude & $2.89 \%$ & $2.62 \%$ & $0.43 \%$ & $0.39 \%$ & $4.73 \%$ & $-0.07 \%$ \\
\hline
\end{tabular}

spread, the rf signal delay due to the cable length error, the frequency, amplitude, and phase control error on different harmonic modes and in different kicker cavities and groups and so on. These errors may lead to residual distortion which will further enlarge the emittance growth.

Table IV summarizes the additional emittance growth due to $+/-5 \%$ deviations in the betatron phase advance, the voltage amplitude in one of the kickers and the rf phase delay between two kickers. In practice, it is easy enough to control the errors within 5\% deviation but here it is used to show the tolerance estimate. Here the errors are calculated from the largest emittance deviation during the 10-turn circulation, and the emittance growth is relative to the ideal situation for each kicking scheme and for each error source. The least-modes scheme is more sensitive to the betatron phase error and the voltage amplitude error. The flat-top scheme is more sensitive to the time delay error but is still within acceptable limits. In the actual operation, these errors need to be carefully controlled to meet the beam dynamics requirement.

A suitable kicker waveform should appropriately balance the emittance preservation performance and the engineering cost. Further options to optimize the kicker scheme are being studied, including replacing the predistortion and postdistortion kickers with single mode cavities, or reintroducing higher harmonics to the least-modes scheme to get a more uniform kick. In this paper, the kicker device we will discuss in the following sections is based on the flat-top waveform for a 10-turn recirculation with 10 Harmonic modes and a DC offset. The cavity design and harmonic distributions for other waveform schemes can be simplified from this version.

\section{RF KICKER CAVITY DESIGN}

Devices with the capabilities to generate such harmonic modes have been explored, fabricated and tested in the past [10-13], and for transverse kick application a traditional stripline kicker with a compact structure was developed [13]. However as a traveling wave device significant power is required, most of which ends up in the matching load. To give a 1 mrad kick angle, several tens of $\mathrm{kW}$ power would be needed [16]. With low efficiency high power feedthroughs and loads are needed. If a larger kick angle is required, this type of kicker is even more inefficient and impractical.
Another method to generate such a transverse kick is to introduce a skew beam pipe to a longitudinal kicker device [10], thus the bunch can be kicked by the transverse component of the longitudinal kick. However, the longitudinal component still has a non-negligible effect on the bunch, which will reduce the kicker efficiency and may result in longitudinal instability in the circulation ring.

Our approach to build a transverse harmonic kicker is to use quarter wave resonators (QWRs). A QWR consists of a quarter wavelength transmission line shorted at one end and open-circuit at the end where the beam passes through. The high impedance in the end gap can be used to build up high voltages needed for particle acceleration [18,19], deflecting, and crabbing [20]. In this case the capacitance at the open-circuit end produces the transverse fields for kicking the beam, as shown in Fig. 11, but also slightly changes the frequency of each mode and must be compensated. The fundamental frequency in the cavity is designed to match the kick repetition frequency $(47.63 \mathrm{MHz})$, higher order modes in this cavity are at nearly odd multiples of the fundamental frequency, and can be made exact harmonic frequencies by some tuning methods. Since the QWR cannot support even harmonics of the fundamental, additional cavities are needed to obtain the full set of modes. Four quarter wavelength resonators are designed and optimized in order to generate 10 harmonic modes for a flat-top kick profile as a proof-of-principle device for a 10 turn recirculation cooler ring [14,21-23], which is illustrated in Fig. 12.

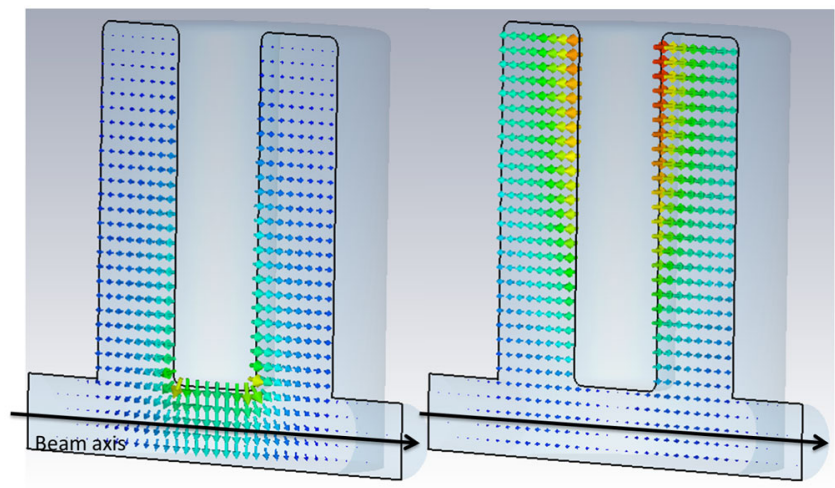

FIG. 11. The distribution of electric field (left) and magnetic field (right) in the deflecting QWR. 


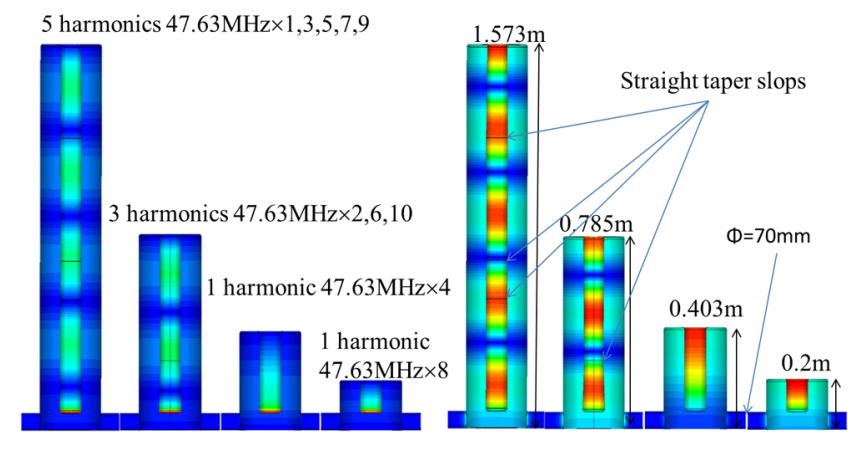

FIG. 12. 10 harmonic modes in a four-cavity system with the highest harmonic electric field (left) and magnetic field (right) distribution shown in each cavity.

The relationship between cavity number $M$ and maximum harmonic number $N$ can be supported as:

$$
2^{M}-1 \leq N
$$

With four cavities, the maximum number of useful harmonic modes that can be obtained is 15 , and with five cavities, up to 31 harmonic modes can be generated. Depending on the number of harmonic modes needed, the total cavity number and the harmonic mode distribution within one cavity can be adjusted. From this point of view, the least-modes waveform has a great advantage by using only half the number of harmonic modes. In this 10 turn recirculation example, only three cavities would be needed to generate five modes with the distribution proportion of $3: 1: 1$. It would also be possible to use five single frequency cavities to generate the kick waveform. A single frequency cavity is much easier to build and tune and could be more compact, for example by using a folded structure [9]. It would also be possible to use superconducting cavities to get a much higher kick voltage. However for larger numbers of modes needed for more recirculation passes this would be less practical.

When an electron bunch passes through the multiple cavity gaps, it will receive an integrated kicking voltage from all these harmonic modes. It is deflected primarily by the transverse electric field, with a very small reverse deflection by the transverse magnetic field. For relativistic electron bunches traveling in the z-direction and deflected in the x-direction, the transverse kick voltage of the $n$th harmonic is calculated from:

$$
\begin{aligned}
V_{t n}= & \int_{-\infty}^{\infty}\left[E_{x n}(z) \cos \left(2 \pi z / \lambda_{n}\right)\right. \\
& \left.+c B_{y n}(z) \sin \left(2 \pi z / \lambda_{n}\right)\right] d z .
\end{aligned}
$$

Here $\lambda_{n}$ is the wavelength of the $n$th harmonic. To give a $1 \mathrm{mrad}$ kick angle for $55 \mathrm{MeV}$ electron bunches in the JLEIC cooler ring, the required kick voltage is $55 \mathrm{kV}$, which could be easily achieved by a normal conducting copper cavity. At higher voltages the main concern with normal conducting cavities would be the thermal load in continuous wave operation. Water cooling is needed to remove the heat and stabilize the frequency. However, above a certain thermal load, it is no longer possible to remove the heat from the cavity wall without losing performance or reliability [24]. To minimize the heat load these cavities are optimized to get a maximum transverse shunt impedance. With the high shunt impedance of this type of cavity, the total $\mathrm{rf}$ power dissipated on the cavity wall is less than $100 \mathrm{~W}$ [22], two to three orders of magnitude lower than a stripline type kicker for a given electron energy and kick angle [16]. In this lower power situation, water cooling may not even be necessary but may be useful to stabilize the frequency.

\section{MULTIPOLE FIELD ANALYSIS}

The asymmetric cylindrical structure will produce higher order multipole field components, such as quadrupole, sextupole and octopole, which could lead to emittance growth. Unlike the double quarter wavelength cavity [20] and TEM-like (transverse electric and magnetic field) deflecting cavities [25], the beam axis in this kicker cavity is not in the structure symmetric plane, which will result in a more nonuniform deflection. If the beam axis is in the $\mathrm{z}$-direction, and bunches will be kicked in the x-direction, the field within the beam pipe varies across the beam aperture in both $\mathrm{x}$ and $\mathrm{y}$ directions. The normalized transverse voltage variation are shown in Fig. 13. In this figure, the increasing positive offset in the $\mathrm{x}$-direction means closer to the inner conductor center electrode where the fields are stronger.

For fully relativistic particles the transverse rf kick strength can be expressed as a magnetic kick of the same strength, and the transverse momentum imparted by the higher order multipole field components can be determined by using Panofsky Wenzel theorem or by using the Lorentz

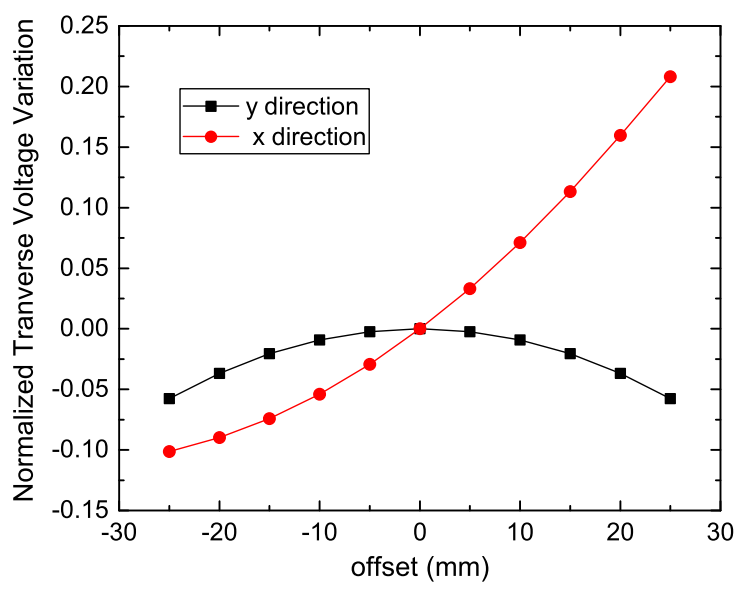

FIG. 13. Normalized transverse voltage variations in $\mathrm{x}$ any $\mathrm{y}$ direction, offset $=0$ is the beam line center. 
TABLE V. Multipole components for the 5 odd harmonics.

\begin{tabular}{|c|c|c|c|c|c|c|}
\hline & 1 & 3 & 5 & 7 & 9 & Units \\
\hline Vz & 0 & 0 & 0 & 0 & 0 & MV \\
\hline $\mathrm{Vt}$ & 1 & 1 & 1 & 1 & 1 & MV \\
\hline b1 & 3.336 & 3.338 & 3.34 & 3.338 & 3.336 & $\mathrm{mTm}$ \\
\hline b2 & 23.72 & 23.53 & 23.14 & 22.65 & 22.28 & $\mathrm{mT}$ \\
\hline b3 & 334.0 & 335.0 & 333.8 & 333.9 & 340.3 & $\mathrm{mT} / \mathrm{m}$ \\
\hline b4 & 120.0 & 127.5 & 236.0 & 404.2 & 642.4 & $\mathrm{mT} / \mathrm{m} 2$ \\
\hline b5 & 17360 & 15420 & 16360 & 17820 & 19970 & $\mathrm{mT} / \mathrm{m} 3$ \\
\hline
\end{tabular}

force [26-28]. In this case the higher order multipole components of the 5 odd harmonic modes are shown in Table V. In this calculation, all the higher order multipole components are normalized to a transverse voltage $\left(V_{t}\right)$ of $1 \mathrm{MV}$.

Here $b_{1}$ is the dipole component which produces to the transverse kick seen by the particles. $b_{2}$ is the quadrupole multipole component, which will introduce horizontal and vertical tune shift in the ring, but has no effect on the transverse emittance. $b_{3}$ is the sextupole component which could produce chromaticity shift and transverse emittance growth. The emittance growth due to the sextupole was simulated in ELEGANT with zero length deflectors, and was shown to have no significant effect on the transverse emittance during the recirculation, the tracking result is as good as shown in Fig. 8. Obvious chromaticity shift and transverse emittance growth are seen if the sextupole coefficient is artificially increased by three orders of magnitude in the simulation. Higher multipole component like octopole and above are much weaker and their contributions to transverse beam dynamics are negligible.

\section{MULTICAVITY KICK-DRIFT TRACKING}

Unlike a stripline rf kicker, in which all harmonic modes can be combined at a single device, the resonant cavity kickers in our design provide the kick at four different locations, with drift in between, as shown in Fig. 14. The drifts between the cavities will introduce additional transverse displacement, and the beam optics design needs to carefully cancel the displacement.

In the simulation, we continue to approximate the kick in each cavity with a series of zero length deflectors, but with a drift space between each pair of two cavities. For the best cancellation effect, the 180 degree phase advance should be enforced between each pair of two identical cavities; the phase advance between two kicker systems (from K24 to K11) should be 180 degrees minus the phase advance over a kicker length of drift (from K21 to K24), i.e. $(\pi-\varphi)$ in Fig. 14. For this simplified kick-drift model for nonmagnetized electron bunches, the betatron phase advance is given by:

$$
\varphi=\int_{0}^{L} d s / \beta(s)
$$

Here $L$ is the total drift space $(L 1+L 2+L 3)$, and $\beta(s)$ is the betatron amplitude function. With this adjustment, the residual kick was totally cancelled, and the tracking result shows that the emittance growth is almost the same as the result of the single kick model shown in Fig. 8. Further

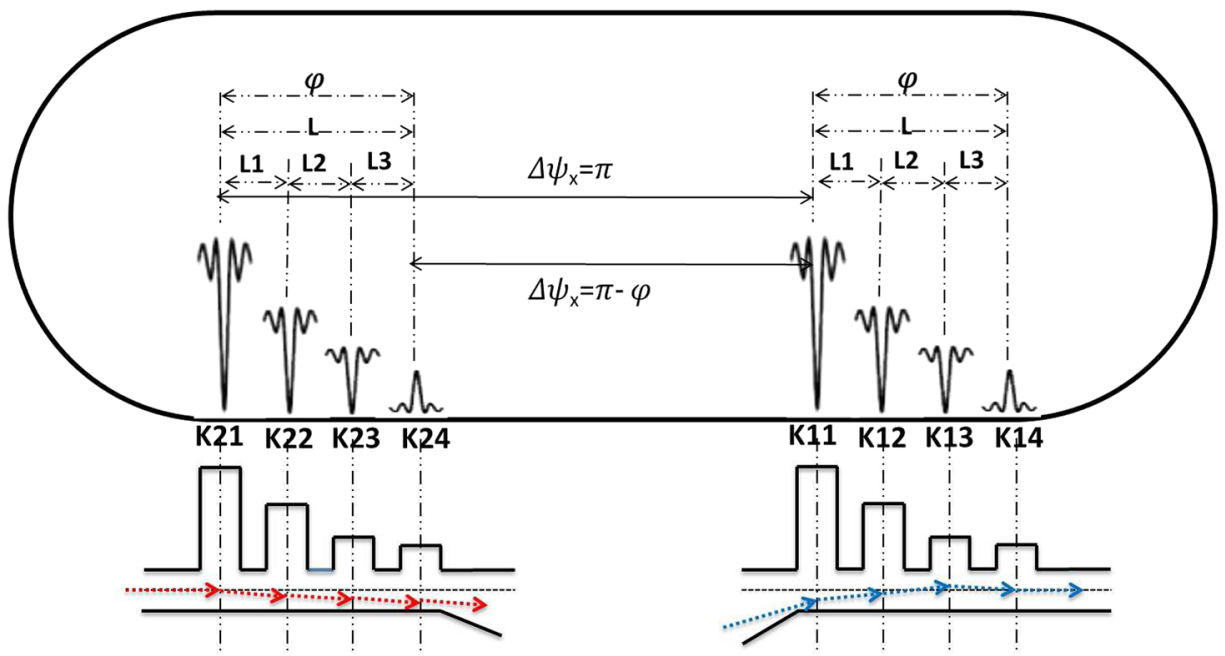

FIG. 14. Multicavity kick-drift tracking scheme with off-axis injection and extraction for flat-top waveform (the kick voltage is reversed at the last cavity as it is mainly used for curvature correction). 
beam dynamics tracking with a magnetized electron bunch and full 3D field maps of the kicker cavities are planned.

\section{OFF-AXIS INJECTION AND EXTRACTION}

Another concern caused by the multicavity scheme is the off-axis injection and extraction which is shown in Fig. 14.

When the bunch is injected into the ring, to make sure the bunch is on the design orbit, a small offset is needed. This offset is determined by the drift space between cavities and the kick voltage distribution in the four cavities. For the flat-top waveform, with a $0.5 \mathrm{~m}$ drift space between two cavities, the offset is about $0.2 \mathrm{~mm}$, with the same situation at extraction. Due to nonuniform kick voltage across the beam pipe shown in Fig. 13, at $0.2 \mathrm{~mm}$ offset in the $\mathrm{x}$-direction, the kick voltage change is about $0.08 \%$, which should not be a large problem. Further confirmation simulations with 3D field maps are planned.

\section{CONCLUSIONS}

We compared several schemes to synthesize the harmonic rf kicker waveform for the JLEIC CCR injection and extraction, with multiple-particle tracking using ELEGANT. By separating the injection and extraction kickers with 180 degrees betatron phase advance along the recirculation path, the disturbance for the recirculating bunches from the residual waveform of the two kickers can be cancelled locally, so all the schemes can maintain injection beam emittance during the recirculation. The flattop waveform has the minimum emittance growth during the injection and extraction due to its uniform kick within the bunch length. Without significant compromise in performance, the least-modes waveform reduces the number of harmonic modes by half, simplifying the kicker with less cavities and fewer harmonic modes in each cavity. For applications with higher kicking voltage, especially when superconducting cavities are required, the least-modes scheme makes it possible to use 5 single mode cavities to achieve such a 10-turn recirculation kicker pulse. Four QWR based deflecting cavities are being developed to generate 10 harmonic modes required in the flat-top scheme. With high transverse shunt impedance, the sum of the simulated rf wall loss in all four cavities is less than $100 \mathrm{~W}$ for a kicking voltage of $55 \mathrm{kV}$. Analysis shows the encouraging result that the quadrupole and sextupole field components have no significant effect on the beam quality and the higher order multipole fields have negligible influence on the beam transverse emittance. The multicavity scheme tracking with a simplified kick-drift model shows encouraging results after the adjustment of the relative betatron phase between two kickers. The injection and extraction offset due to this multicavity scheme is also not a problem for the simple beam optics. Further beam dynamics tracking with magnetized electron bunches and real 3D cavity field maps is planned. A half scale cavity made of copper is being fabricated and bench measurements will be carried out in the summer of 2016.

\section{ACKNOWLEDGMENTS}

Work supported by Jefferson Science Associates, LLC under U.S. DOE. Contract No. DE-AC05-06OR23177. The authors acknowledge the useful previous works on the harmonic kicker concept, and especially thank Amy Sy for the helpful discussion on the kicker waveform schemes and ELEGANT tracking, and Subashini de Silva for the help on the multipole field coefficient calculation.

[1] S. Abeyratne et al., Science requirements and conceptual design for a polarized medium energy electron-ion collider at Jefferson lab, arXiv:1209.0757.

[2] S. Abeyratne et al., MEIC design summary, arXiv:1504.07961.

[3] Y. Derbenev, J. Musson, and Y. Zhang, Electron cooling for a high luminosity electron-ion collider, in Proceedings of COOL'07 Workshop on Beam Cooling and Related Topics, Bad Kreuznach, Germany (2007).

[4] Y. Zhang, Y. Derbenev, D. Douglas, A. Hutton, A. Kimber, R. Li, E. Nissen, C. Tennant, and H. Zhang, Advance in MEIC cooling studies, in Proceedings of COOL'13 International Workshop on Beam Cooling and Related Topics, Murren, Switzerland (JACoW, Murren, 2013).

[5] R. A. Rimmer, J. Guo, J. Henry, Y. Huang, H. Wang, and S. Wang, RF system requirements for a Medium-Energy Electron-Ion Collider (MEIC) at JLab, in Proceedings of the 6th International Particle Accelerator Conference (IPAC2015), Richmond, VA, USA (JACoW, Richmond, 2015).

[6] V. Kempson, J. A. Dobbing, G. Duller, N. Hauge, G. Hilleke, and C. Hansen, Pulsed magnets and pulser units for the booster and storage ring of the Diamond Light Source, in Proceedings of the 10th European Particle Accelerator Conference, Edinburgh, Scotland, 2006 (EPS-AG, Edinburgh, Scotland, 2006).

[7] V. Shiltsev, Beam-beam kicker for superfast bunch handling, Nucl. Instrum. Methods Phys. Res., Sect. A 374, 137 (1996).

[8] G. Gollin, Speculations about a Fourier series kicker for the TESLA damping ring, in Proceedings of Arlington Linear Collider Workshop, University of Texas at Arlington (2003).

[9] E. Nissen, A. Hutton, and A. Kimber, A harmonic kicker scheme for the circulator cooler ring in the medium energy electron ion collider, in Proceedings of the 4th International Particle Accelerator Conference, IPAC2013, Shanghai, China, 2013 (JACoW, Shanghai, China, 2013).

[10] B. Roberts, R. R. Mammei, M. Poelker, and J. L. McCarter, Compact noninvasive electron bunch-length monitor, Phys. Rev. ST Accel. Beams 15, 122802 (2012).

[11] Y. Iwashita, Multi-Harmonic impulse cavity, in Proceedings of the 18th Particle Accelerator Conference, New York, 1999 (IEEE, New York, 1999). 
[12] F. Caspers, H. Haseroth, and J. Knott, High gradients by simultaneous multifrequency operation of rf structure, in Proceedings of the 1987 Particle Accelerator Conference (IEEE, Washington, DC, 1987).

[13] W. Barry, J. Byrd, J. Corlett, M. Fahmie, J. Johnson, G. Lambertson, M. Nyman, J. Fox, and D. Teytelman, Design of the PEP-II transverse coupled-bunch feedback system, in Proceedings of the Particle Accelerator Conference, Dallas, TX, 1995 (IEEE, New York, 1995).

[14] Y. Huang, R. Rimmer, H. Wang, S. Wang, and J. Guo, Ultrafast harmonic resonant kicker design for the MEIC electron circular cooler ring, in Proceedings of 56th ICFA Advanced Beam Dynamics Workshop on Energy Recovery Linacs (ERL2015), Stony Brook, NY, USA (JACoW, Stony Brook, 2015).

[15] A. Sy, A. Kimber, and J. Musson, Development of an ultrafast rf kicker for an ERL-based electron cooler, in Proceedings of COOL15 Workshop on Beam Cooling and Related Topics, Newport News, VA, USA (JACoW, Newport News, 2015).

[16] J. Guo and H. Wang, Harmonic stripline kicker for MEIC bunched beam cooler, in Proceedings of COOL15 Workshop on Beam Cooling and Related Topics,Newport News, VA, USA (JACoW, Newport News, 2015).

[17] M. Borland et al., Elegant: A flexible SDDS-compliant code for accelerator simulation, Argonne National Lab, IL, US, 2000 (unpublished).

[18] S. Belomestnykh, I. Ben-Zvi, C. Boulware et al., Superconducting $112 \mathrm{MHz}$ QWR electron gun, in Proceedings of 15th International Conference on RF Superconductivity, Chicago, IL, USA (2011).

[19] D. Naik and I. Ben-Zvi, Suppressing multipacting in a 56 $\mathrm{MHz}$ quarter wave resonator, Phys. Rev. ST Accel. Beams 13, 052001 (2010).

[20] B. Xiao, L. Alberty, S. Belomestnykh, I. Ben-Zvi, R. Calaga, C. Cullen, O. Capatina, L. Hammons, Z. Li, C. Marques et al., Design, prototyping, and testing of a compact superconducting double quarter wave crab cavity, Phys. Rev. ST Accel. Beams 18, 041004 (2015).

[21] Y. Huang, H. Wang, R. A. Rimmer, and S. Wang, Taper and tuner scheme of a multi-frequency cavity for the fast kicker resonator in MEIC electron circular cooler ring, in Proceedings of COOL15 Workshop on Beam Cooling and Related Topics, Newport News, VA, USA (JACoW, Newport News, 2015).

[22] Y. Huang, H. Wang, R. A. Rimmer, and S. Wang, Harmonic resonant kicker design for the MEIC electron circular cooler ring, in Proceedings of the 6th International Particle Accelerator Conference, Richmond, VA, USA (JACoW, Richmond, 2015).

[23] Y. Huang, H. Wang, R. A. Rimmer, and S. Wang, Beam dynamics analysis for the ultra-fast kicker in circular cooler ring of JLEIC, in Proceedings of the 7th International Particle Accelerator Conference, Busan, Korea (JACoW, Busan, 2016).

[24] H. Podlech, Superconducting versus normal conducting cavities, arXiv:1303.6552.

[25] S. U. De Silva and J. R. Delayen, Design evolution and properties of superconducting parallel-bar rf-dipole deflecting and crabbing cavities, Phys. Rev. ST Accel. Beams 16, 012004 (2013).

[26] S. U. D. Silva and J. R. Delayen, Multipole field effects for the superconduting parallel-bar/rf-dipole deflecting/ crabbing cavities, in Proceedings of LINAC2012, Tel-Aviv, Israel (JACoW, Tel-Aviv, 2012).

[27] M. S. Chae, J. H. Hong, Y. W. Parc, I. S. Ko, S. J. Park, H. J. Qian, W. H. Huang, and C.X. Tang, Emittance growth due to multipole transverse magnetic modes in an rf gun, Phys. Rev. ST Accel. Beams 14, 104203 (2011).

[28] J. B. Garcia, R. Calaga, R. De Maria, M. Giovannozzi, A. Grudiev, R. Tomás et al., Study of multipolar rf kicks from the main deflecting mode in compact crab cavities for LHC, in Proceedings of the 3rd International Particle Accelerator Conference, New Orleans, LA, 2012 (IEEE, Piscataway, NJ, 2012). 